\title{
SMALL BOWEL DIVERTICULOSIS WITH JEJUNAL ATRESIA
}

\author{
S. Srinivas ${ }^{1}$, K. Ramesh Reddy², K. Lavanya ${ }^{3}$
}

\section{HOW TO CITE THIS ARTICLE:}

S. Srinivas, K. Ramesh Reddy, K. Lavanya. "Small Bowel Diverticulosis with Jejunal Atresia”. Journal of Evolution of Medical and Dental Sciences 2015; Vol. 4, Issue 56, July 13; Page: 9849-9851,

DOI: $10.14260 /$ jemds/2015/1423

CASE REPORT: A 24 years old primigravida had presented to us in third trimester with an antenatal ultrasound diagnosis of intestinal obstruction in the fetus and polyhydramnios. The parents were counseled and the obstetrician was advised to conduct the delivery as per obstetric indication, the patient delivered at $36 \mathrm{wks}$ by an emergency LSCS. The baby was $1.8 \mathrm{Kgs}$ in weight and had an APGAR score of 9 and 10 at 1 and 5 minutes of life. Baby had significant bilious aspirate and did not pass meconium, it did not have dysmorphic features or any other associated anomalies, abdomen was soft and no masses were palpable. X ray abdomen revealed three gas bubbles with distal paucity of gas (Triple bubble sign). With the above findings a diagnosis of jejunal atresia was made and a laparotomy was planned on day 2 of life.

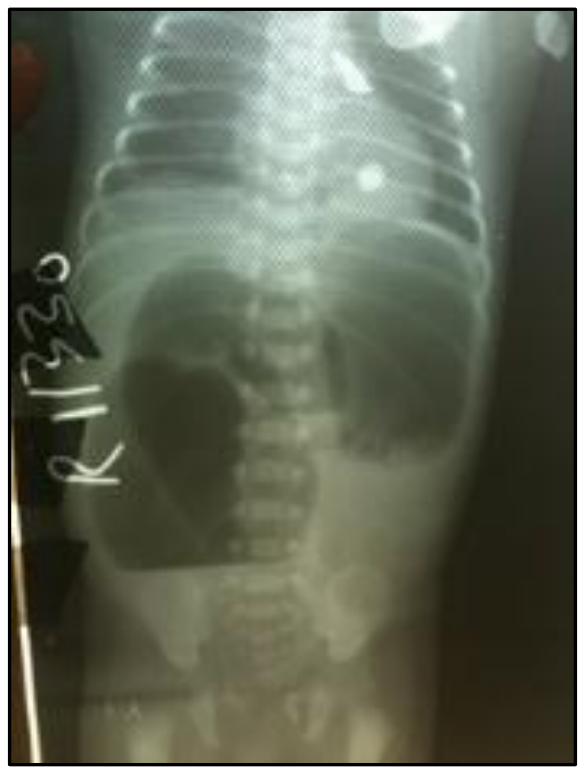

\section{Fig. 1: X-ray abdomen showing triple bubble sign}

At laparotomy a proximal jejunal atresia was found about $20 \mathrm{cms}$ from the DJ flexure. It was a type $3 \mathrm{~A}$ atresia with distal unused bowel. There were multiple diverticula on the mesenteric side of the unused small bowel, large intestine was unused and other wise was normal without diverticula.

Inspissated meconium plugs in distal bowel were washed out with warm saline. During this procedure an accidental perforation was made in the jejunum about $10 \mathrm{cms}$ from atretic site, this segment was resected and an end to back jejunal anastomosis was made with 5'o vicryl. Post operatively child recovered well and was started on antibiotics; TPN was started on 2 POD, baby passed motion on the 12 POD and feeds were started on 14 POD. After observation child was discharged on $20^{\text {th }}$ POD. The child is being followed up on a monthly basis and is one year old now and has attained all his milestones normally till date. 


\section{CASE REPORT}

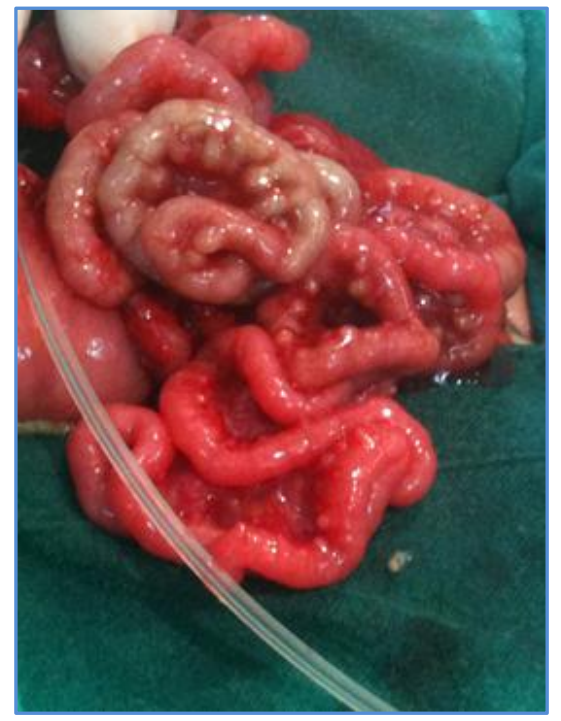

Fig. 2: Intraoperative photograph showing jejunal atresia with multiple diverticulae on the mesenteric side

The resected segment of unused jejunum was evaluated by pathologist. Macroscopically there were diverticula of sizes 1-2 $\mathrm{mm}$ on the mesenteric side, microscopy revealed absent or defective longitudinal muscle and irregular arrangement of circular muscle in these areas of diverticula.

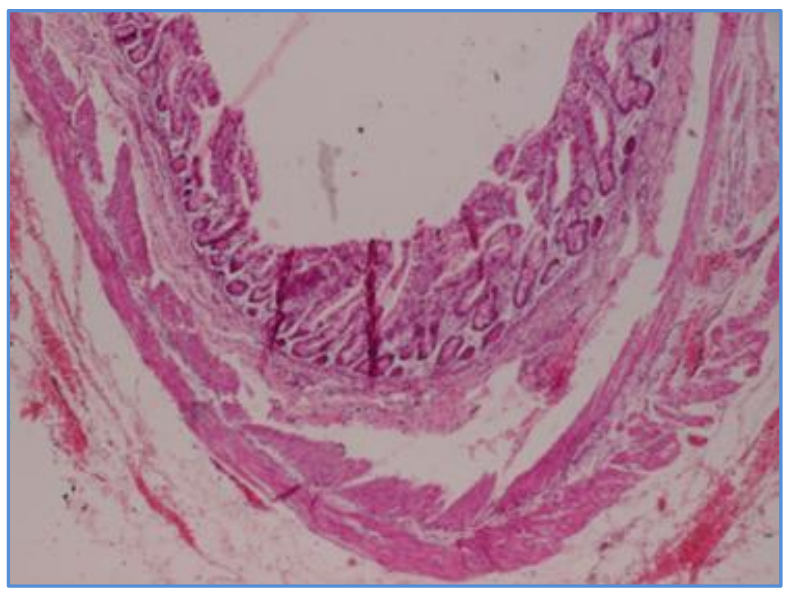

Fig. 3: Histopathological picture of jejunal diverticulae showing attenuated mucosa, submucosa and smooth muscle

DISCUSSION: MU Shenoy et al(1) had reported a similar case with multiple jejunal atresias with diverticulae proximal and distal to atretic segments. They demonstrated focal absence of intestinal musculature in these areas. The pathogenesis of absence of intestinal musculature is not known. Mc Carthy et al(2) based on the fact that a 7 to $40 \mathrm{~mm}$ embryo has multiple diverticula which regress proposed an embryological etiology. Alawadhi et al(3) had proposed that this process of regression of diverticula if fails leads to segmental absence of musculature. Emanuel et al(4) had stated a genetic cause for absence of intestinal musculature. 


\section{Husain et al(5) suggested classifying segmental absence of intestinal musculature into two} groups:

1. Primary in which there were no pathologic findings in the remaining layers of small bowel other than superimposed perforation or intussusception.

2. Secondary in which there may be ischemic necrosis, fibrosis, calcification, or chronic inflammation in the remaining layers of intestine. These findings may be caused by vascular accident or trauma.

Alvarez et $\mathrm{al}^{(6)}$ had reported a case of ileal atresia with segmental absence of intestinal musculature which led to perforation and meconium peritonitis. Sections from atretic portion also showed focal absence of muscle layers. They believed that vascular accident explains both atresia and absence of musculature.

The exact etiology of atresia with diverticulae remains uncertain, segmental absence of intestinal musculature may be the cause of these diverticulae and vascular accident may be the possible cause of this association of atresia and multiple diverticulae.

\section{REFERENCES:}

1. M. U. Shenoy, K. Robson, N. Broderic, and L. Kpila: Congenital small bowel diverticulosis and intestinal atresia: A rare association. J pediatric surg, vol 35: 636-637, 2000.

2. McCarthy DW, Qualman S, Besner GE: Absence of intestinal musculature: Anatomic evidence of an embryonic origin of lesion. J Pedtaric surg. 29: 1476-1478, 1994.

3. Alawadhi A, Chou S, Capenter B: Segmental agenesis of intestinal muscularis: A case report. J Pediatric surgery 24: 1089-1090, 1989.

4. Emanuel B, Gault J, Sanson J: Neonatal intestinal obstruction due to absence of intestinal musculature: A new entity. J pediatric surgery 2: 332, 1967.

5. Husain AN, Hong HY, Gooneratne S: Segmental absence of intestinal musculature. Pediatric pathology 12: 407-415, 1992.

6. Alvarez SP, Greco MA, Geneiser NB: Small intestinal atresia and segmental absence of muscle coat. Human pathology 13: 948-951, 1982.

\section{AUTHORS:}

1. S. Srinivas

2. K. Ramesh Reddy

3. K. Lavanya

\section{PARTICULARS OF CONTRIBUTORS:}

1. Assistant Professor, Department of Paediatric Surgery, Niloufer Hospital, Osmania Medical College, Hyderabad.

2. Professor, Department of Paediatric Surgery, Niloufer Hospital, Osmania Medical College, Hyderabad.
3. Assistant Professor, Department of Paediatric Surgery, Niloufer Hospital, Osmania Medical College, Hyderabad.

NAME ADDRESS EMAIL ID OF THE CORRESPONDING AUTHOR:

Dr. S. Srinivas, \# 3-4-771/401, Thakur Residence, Barkatpura, Hyderabad-500027.

E-mail: shantisrinivas@gmail.com

Date of Submission: 16/06/2015. Date of Peer Review: 17/06/2015. Date of Acceptance: 04/07/2015. Date of Publishing: 13/07/2015. 